\title{
Somatotipo y Aptitud Física en Árbitros Asistentes de Fútbol
}

\author{
Somatotype and Physical Fitness of the Assistant Referees in Soccer
}

Alberto Inácio da Silva

SILVA, A. I. Somatotipo y aptitud física en árbitros asistentes de fútbol. Int. J. Morphol., 29(3):792-798, 2011.

RESUMEN: El objetivo de este estudio fue analizar el somatotipo de los árbitros asistentes y verificar su correlación con el rendimiento físico de los mismos durante los tests de la FIFA. La población de este estudio fue constituida por árbitros de la Confederación Brasileña de Fútbol (CBF) y de la Federación Paranaense de Fútbol (FPF) que se presentaron a la Comisión de Evaluación Física de la FPF, para someterse a las pruebas de aptitud física. La muestra fue constituida por 11 árbitros de la CBF, considerados de elite en Brasil, y 34 árbitros de la FPF que actúan a nivel regional en la provincia de Paraná. Para determinar la composición corporal se utilizaron: nueve pliegues cutáneos, nueve circunferencias corporales y cuatro diámetros óseos. Para evaluar las variables relacionadas con la aptitud física se utilizó la batería de tests físicos de la FIFA, compuesta de las siguientes pruebas para la evaluación de los árbitros asistentes: dos carreras de 50 metros y una carrera de 12 minutos. El análisis de los datos de la composición corporal, indicaron que el porcentaje de grasa de los árbitros asistentes de la CBF fue de 16,74 $\pm 3,71 \%$, y que los árbitros asistentes de la FPF presentaron 17,09 $\pm 4,38$. Esta diferencia no fue estadísticamente significativa ( $>>0,05$ ). Con relación al somatotipo, los árbitros asistentes de la CBF fueron clasificados como meso-endomorfo y los árbitros asistente de la FPF de endo-mesomorfo, o sea, en el grupo de asistente de la CBF predominó el componente muscular sobre el adiposo. La diferencia entre los somatotipos fue estadísticamente significativa (DDS=2,22). Los árbitros asistentes de la CBF fueron más veloces que los árbitros de la FPF, en el test de velocidad, siendo la diferencia entre los resultados estadisticamente significativa $(\mathrm{p}=0,0001)$. Los árbitros asistentes realizan esfuerzos menores que el árbitro principal durante el partido, pero sus rendimientos en los tests físicos son semejantes al juez central.

PALABRAS CLAVE: Árbitros; Fútbol; Somatotipo.

\section{INTRODUCCIÓN}

Un partido de fútbol para ser conducido con eficiencia debe tener un equipo compuesto por cuatro árbitros. Uno actuará como árbitro principal, dos de ellos serán sus asistentes que llevarán las banderolas y el cuarto que se ubica entre las bancas de ambos equipos. Sus funciones dentro del campo de juego están definidas en el reglamento de fútbol. La regla cinco describe la actuación del árbitro y la seis las funciones de los asistentes (FIFA, 2010). El surgimiento del fútbol como se practica hoy data del año 1863 y es en el año 1868 cuando aparece la figura del árbitro de fútbol. Sus asistentes son incorporados en este deporte en 1891 (Duarte, 1997).

Debido a la importancia del árbitro para el fútbol, la comunidad científica pasó a estudiarlo para fundamentar su preparación física y mental. Mientras trabajos de cuño científico, involucrando árbitros de fútbol son muy recientes y escasos, si tomamos como referencia los estudios involucrando jugadores de fútbol (Castagna et al., 2002;
Weston et al. 2004; Da Silva, 2005). Entre los pocos estudios involucrando el arbitraje de fútbol la mayoría son direccionados al árbitro principal, pues se reconoció que el árbitro es también una de las personas que pueden interferir en el resultado de un partido, pues una decisión precipitada o equivocada, puede retirar de la competición un equipo que invirtió millones de dólares en la compra y preparación de sus jugadores, restando a este apenas lamentar. Toda acción llevada a cabo por los humanos tiene el error implícito. Es una característica de la repentización del juego y las desiciones arbitrales. Pero con el crecimiento del fútbol, principalmente con la valoración de la Copa del mundo organizada por la FIFA (Fédération Internacionale of Football Association) y los campeonatos nacionales, los grandes eventos futbolisticos pasaron a ser transmitidos directamente desde las plazas deportivas y la televisión con su tecnología detectaron que también las acciones de los árbitros asistentes estaban interferiendo en los resultados, principalmente 
en la validación de los goles. Por lo tanto muy recientemente, empezaron a surgir estudios académicos dirigidos a los asistentes y estos son muy escasos (Mallo et al., 2008).

El primer estudio involucrando los árbitros asistentes del que tenemos conocimiento fue desarrollado en Brasil y describia el gasto energético de los asistentes durante el partido. Despúes surgieron las descripciones de sus acciones motoras durante el partido (Krustrup et al. 2002; Da Silva \& Fernandez, 2003), y su deshidratación durante el partido que también ya fue estudiada (Da Silva \& RodriguezAñez, 2003). El más reciente estudio involucrando árbitros asistentes describe la correlación de sus acciones durante el partido y los tests físico de la FIFA.

El somatotipo de los árbitros asistentes no fue discutido a nivel internacional. Datos referentes al somatotipo en árbitros de fútbol son muy escasos en la literatura científica, pero el uso en todo el mundo de la técnica antropométrica del somatotipo está muy extendida en un intento de verificar la relación entre el desarrollo físico y el rendimiento deportivo en diferentes disciplinas deportivas (Carter, 1968; Rahmawati et al., 2006). Los estudios brasileños se llevaran a cabo también con el mismo compromiso en algunos deportes (Junior et al., 2006; Queiroga et al., 2005). De este modo, el objetivo de este estudio fue analizar el somatotipo de los árbitros asistentes de elite y verificar su correlación con el rendimiento físico de los mismos durante los tests físicos determinados por la FIFA.

\section{MATERIAL Y MÉTODO}

La población de este estudio fue constituida por árbitros de la Confederación Brasileña de Fútbol (CBF) y de la Federación Paranaense de Fútbol (FPF) que se presentaron a la Comisión de Evaluación Física de la FPF, para someterse a las pruebas de aptitud física. La muestra fue constituida por 11 árbitros de la CBF, árbitros de elite de Brasil, y 34 árbitros de la FPF, que actuan a nivel regional en la provincia de Paraná. Todas las evaluaciones fueron realizadas en un único día, por la mañana.

Los procedimientos adoptados en este estudio están de acuerdo con las técnicas adecuadas descritas en la literatura y no implican riesgos o perjuicios a los evaluados. Antes del inicio de la recolección de los datos, fueron informados los propósitos y procedimientos técnicos que serian adoptados, enfocando los objetivos y beneficios de la investigación para los participantes, a través de una reunión del responsable de la investigación, junto con el preparador físico de los árbitros, así que como de toda la muestra de estudio.
En esta reunión fue garantizada la libertad de asistencia de participación en el estudio por parte de los árbitros antes y durante las evaluaciones.

Para la evaluación de la composición corporal fueron medidos: nueve pliegues cutáneos (subescapular, tríceps, bíceps, pectoral, axilar promedio, abdomen, suprailíaca, muslo y pantorrilla) utilizando un adipómetro marca Cescorf de fabricación brasileña, con una precisión de medida de 0,1 mm. También se midieron nueve circunferencias corporales (antebrazo, brazo contraído, brazo relajado, tórax, abdomen, cadera, muslo superior, muslo mediano y pantorri1la) utilizando una cinta métrica flexible marca Cescorf, con una graduación de $0,1 \mathrm{~cm}$. A su vez, se realizó la medición de cuatro diámetros óseos (biestiloide, biepicondiliano del húmero, biepicondiliano del fémur y bimaleolar), empleando un antropómetro de metal o calibre óseo, marca Mitutoyo, con una precisión de $0,1 \mathrm{~cm}$. A la evaluación de estas variables antropométricas (pliegues cutáneos, circunferencias corporales y diámetros óseos), siguieron las estandarizaciones propuestas por Harrison et al. (1991), Callaway et al. (1991) y Wilmore et al. (1991).

Se determinó además la masa corporal, la estatura y la edad conforme Ross \& Marfell-Jones (1995). La densidad corporal fue determinada a partir de las variables antropométricas utilizando la ecuación propuesta por Jakson \& Pollock (1978), que utiliza la suma de 7 pliegues cutáneos y dos perímetros y el porcentual de gordura por la ecuación de Siri (1961). La masa de grasa (MG) fue obtenida multiplicando la masa corporal (MC) por la fracción del porcentual de gordura $(\% \mathrm{G}): \mathrm{MG}=\mathrm{MC}$ x $(\% \mathrm{G} / 100)$. Para la determinación de la masa ósea (MO) y de la masa residual (MR), se utilizaron las ecuaciones de Von Döblen y Würch citadas por De Rose et al. (1984) respectivamente. La masa muscular fue obtenida de la siguiente manera: MM $=\mathrm{MC}-(\mathrm{MO}+\mathrm{MR}+\mathrm{MG})$. La evaluación antropométrica fue realizada siempre antes de las pruebas de aptitud física.

Para fines de comparación, los somatotipos fueron clasificados en categorías de acuerdo con Carter (2002). El somatotipo también fue expuesto en un gráfico (somatocarta), desarrollada por Carter \& Heath (1990), donde fueron calculados los valores de las coordenadas $\mathrm{X}$ y Y: $\mathrm{X}=$ ectomorfia - endomorfia; $\mathrm{Y}=2 \mathrm{x}$ mesomorfia (endomorfia + ectomorfia).

Las pruebas físicas utilizadas fueron las recomendadas por la FIFA para la evaluación de aptitud física de los árbitros de fútbol hasta la fecha de este trabajo. La batería de tests fue constituida por: dos carreras de 50 metros y una carrera de 12 minutos (test de Cooper) los árbitros asistentes no son sometidos a las dos carreras de 200 metros. Las 
pruebas fueron aplicadas en la siguiente orden: dos carreras de 50 metros, seguidas por la carrera de 12 minutos.

Los tests fueron aplicados en una pista de atletismo abierta. El tiempo de recuperación durante la prueba anaeróbica no fue inferior a 5 minutos. Siendo que después de la realización de cada prueba, los individuos permanecían caminando hasta el local del próximo test, caracterizando con esto una recuperación de tipo activa. Después de la ejecución del test anaeróbico fue dado un intervalo de 15 minutos hasta ocurrir la aplicación de la carreras de $12 \mathrm{mi}-$ nutos (Test de Cooper). Para la medición de los tiempos se utilizaron cronómetros marca Technos, modelo Cronus. La distancia mínima para que el árbitro fuera considerado apto establecida por la FIFA para la carrera de 12 minutos es de 2.700 metros, para varones. El tiempo máximo para la carrera de 50 metros es de 7,50 segundos, para varones.

Los resultados son expresados como valores promedios con su desviación estándar. Los valores promedios fueron comparados por el test " $\mathrm{t}$ " de Student. Los resultados fueron considerados estadísticamente significativos cuando la probabilidad de la ocurrencia de hipótesis nula fuera menor que 0,05.

La diferencia entre el somatotipo de los dos grupos de árbitros también se analizó por medio de la distancia de dispersión del somatotipo (DDS) (Hebbelinck et al. 1975). DDS permite verificar la distancia entre dos somatotipos, estableciendo que la distancia es estadísticamente significativa cuando la DDS es $>2$.

\section{RESULTADOS}

En la Tabla I se encuentran los resultados de las evaluaciones antropométricas de los árbitros asistentes de la CBF y de la FPF. No fue encontrada ninguna diferencia estadísticamente significativa cuando se compara cada variable entre los dos grupos ( $p>0,05)$.

Con respecto al somatotipo se observó que el grupo estudiado tuvo un somatotipo promedio de: 3,8-3,9-1,9. Cuando se estratificó por la función desempeñada (asistente de la CBF o asistente de la FPF), los resultados promedio para los árbitros asistentes de la CBF los clasificaron como meso-endomorfo $(3,5-4,4-1,9)$, mientras que el grupo de la FPF tenía una característica (endo-mesomorfo). Las características de cada grupo la exhibe la Tabla II. Las Figuras 1 y 2 exhiben las somatocartas con la distribución de somatopuntos registrados por los árbitros asistentes de la CBF y de la FPF.

En la Tabla III se encuentran los resultados referentes a la prueba que mide la velocidad (carrera de 50 metros) de los árbitros asistentes de la CBF y de la FPF. El análisis de los resultados demostró no tener diferencia estadísticamente significativa $(p>0,05)$ entre la primera y segunda carrera de 50 metros realizadas por los árbitros asistentes de la CBF. Mintras entre las carreras de 50 metros de los árbitros asistentes de la FPF hubo diferencia estadísticamente significativa $(\mathrm{p}=0,0109)$.

Los resultados referentes al test de Cooper son exhibidos en la Tabla IV. Cuando se hace la confrontación de los datos obtenidos por los árbitros asistentes de la CBF versus los árbitros asistentes de la FPF, se verifica que no hubo diferencia estadísticamente significativa $(p>0,05)$.

A pesar de que se ha verificado la existencia de dos somatotipos y de que los resultados en los tests físicos fueron diferentes, no fue encontrada ninguna correlación entre el somatotipo y los tests físicos (p>0,05). En la Tabla $\mathrm{V}$ exhibe todos los resultados relativos al análisis de correlación.

Tabla I. Resultado del análisis estadística de la variables antropométricas.

\begin{tabular}{lcc}
\hline Variables & Asistentes CBF $(\mathbf{n}=\mathbf{1 1})$ & Asistentes FPF $(\mathbf{n}=\mathbf{3 4})$ \\
\hline Edad (años) & $30,09 \pm 5,7$ & $30,59 \pm 5,8$ \\
Masa Corporal $(\mathrm{kg})$ & $74,56 \pm 9,0$ & $73,80 \pm 10,5$ \\
Estatura $(\mathrm{cm})$ & $175,0 \pm 5,2$ & $177,6 \pm 6,5$ \\
IMC $\left(\mathrm{kg} / \mathrm{m}^{2}\right)$ & $24,29 \pm 2,11$ & $23,36 \pm 2,94$ \\
Grasa corporal $(\%)$ & $16,74 \pm 3,71$ & $17,09 \pm 4,38$ \\
Peso grasa $(\mathrm{kg})$ & $12,56 \pm 3,51$ & $12,89 \pm 4,66$ \\
Peso óseo $(\mathrm{kg})$ & $10,41 \pm 1,65$ & $10,90 \pm 1,66$ \\
Peso residual $(\mathrm{kg})$ & $17,97 \pm 2,17$ & $17,79 \pm 2,52$ \\
Peso muscular $(\mathrm{kg})$ & $33,63 \pm 3,97$ & $32,22 \pm 3,89$ \\
\hline
\end{tabular}


SILVA, A. I. Somatotipo y aptitud física en árbitros asistentes de fútbol. Int. J. Morphol., 29(3):792-798, 2011.

Tabla II. Características somatotipológicas de los árbitros asistentes.

\begin{tabular}{lcc}
\hline Variables & Asistentes CBF $(\mathbf{n}=\mathbf{1 1})$ & Asistentes $(\mathbf{n}=\mathbf{3 4})$ \\
\hline Endomorfo & $3,5 \pm 1,0$ & $3,6 \pm 1,2$ \\
Mesomorfo & $4,4 \pm 1,1$ & $3,7 \pm 1,6$ \\
Ectomorfo & $1,9 \pm 0,8$ & $2,5 \pm 1,4$ \\
\hline * difiere estadisticamente $(\mathrm{p}<0,05)$.
\end{tabular}

Tabla III. Promedios de las carreras de 50 metros de los árbitros asistentes.

\begin{tabular}{lcccc}
\hline & & \multicolumn{3}{c}{ Carreras } \\
\cline { 3 - 5 } & & Primero & Segundo & Total \\
\hline Asistente CBF & Promedio & $6,64(6,07-7,09)$ & $6,77(6,31-7,30)$ & 6,70 \\
& D. S. & 0,27 & 0,27 & 0,27 \\
& n & 11 & 11 & 22 \\
Asistente FPF & Promedio & $6,92(6,30-7,89)$ & $7,12(6,50-8,10)$ & 7,02 \\
& D. S. & 0,32 & 0,31 & 0,32 \\
& n & 34 & 34 & 68 \\
\hline
\end{tabular}

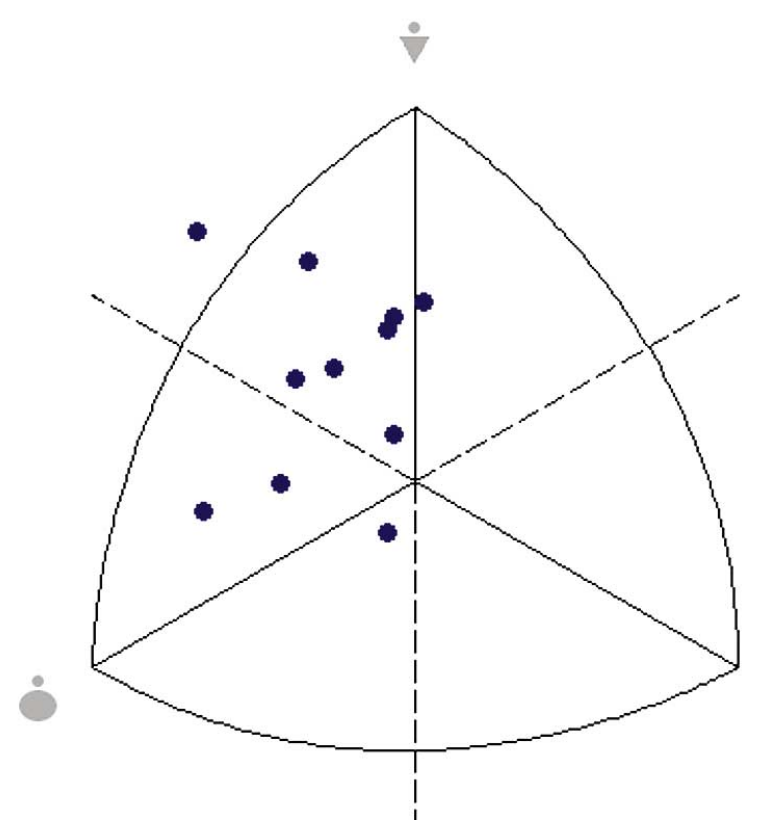

Fig. 1. Somatocarta con distribución de somatopuntos de los árbitros asistentes del fútbol de la CBF.

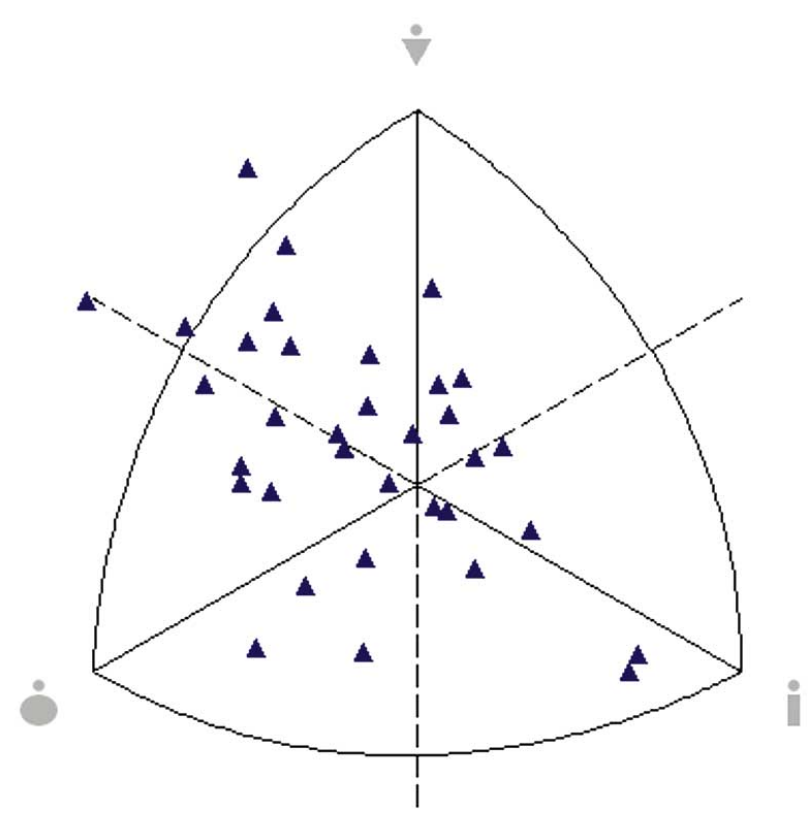

Fig. 2. Somatocarta con distribución de somatopuntos de los árbitros asistentes del fútbol de la FPF.

Tabla IV. Promedio obtenido en el test aeróbico por los árbitros asistentes.

\begin{tabular}{lccc}
\hline & n & Promedio & Desv. \\
\hline Asistentes CBF & 11 & $2859,09(2650-3020)$ & 117,17 \\
Asistentes FPF & 34 & $2854,85(2650-3220)$ & 147,73 \\
\hline
\end{tabular}


Tabla V. Resultados relativos al análisis de correlación entre el somatotipo y los test de aptitud física.

\begin{tabular}{lccccc}
\hline Grupos & Evaluación & Parámetros & ENDO & MESO & ECTO \\
\hline Asistentes CBF & $50 \mathrm{~m}$ & $\mathrm{R}$ & 0,31 & $-0,388$ & 0,0249 \\
& & $p$ & 0,354 & 0,238 & 0,942 \\
& \multirow{3}{*}{ Cooper } & $\mathrm{n}$ & 11 & 11 & 11 \\
& & $\mathrm{R}$ & 0,29 & $-0,213$ & 0,0787 \\
& $p$ & 0,387 & 0,53 & 0,818 \\
Asistentes FPF & \multirow{3}{*}{$50 \mathrm{~m}$} & $\mathrm{n}$ & 11 & 11 & 11 \\
& & $\mathrm{R}$ & 0,147 & $-0,106$ & $-0,0567$ \\
& & $p$ & 0,406 & 0,553 & 0,75 \\
& $\mathrm{n}$ & $\mathrm{n}$ & 34 & 34 & 34 \\
& $\mathrm{Cooper}$ & $\mathrm{R}$ & $-0,138$ & $-0,00595$ & $-0,0471$ \\
& & $p$ & 0,436 & 0,973 & 0,792 \\
& & $\mathrm{n}$ & 34 & 34 & 34 \\
\hline
\end{tabular}

R- Coeficiente de correlación de Pearson; $\mathrm{p}$ - nivel de significación de hipotesis nula; MESOMesomorfo; ENDO - Endomorfo; ECTO - Ectomorfo

\section{DISCUSIÓN}

El porcentaje de grasa estimado en este estudio (Tabla III) fue semejante al valor promedio encontrado por Da Silva \& Rodrigues-Añez (2003) (17,3\%) cuando evaluó a los árbitros de la $\mathrm{CBF}$, los árbitros griegos que presentaron $16,7 \%$ (Rontoyannis et al., 1998) y en uno estudio reciente publicado por Fernández et al. 2008, involucrando árbitros chilenos, estos presentaron 15,4\% de grasa corporal. Entretanto estos estudios fueron realizados con árbitros y no árbitros asistentes, sin embargo el porcentaje fue semejante. Entretanto, en un estudio involucrando árbitros asistentes fue encontrado un valor menor de 14,4\% (Da Silva \& Rodrigues-Añez, 2003) y recientemente de 18,1\% (Da Silva \& Rech, 2008). Estos datos demostraron que el porcentaje de grasa corporal de los árbitros asistentes está aumentando y hay necesidad de someterlos a un programa de ejercicios con asesoramiento nutricional, pues un porcentaje muy elevado puede ser un limitador del rendimiento físico del árbitro durante el partido (Da Silva). Además estudios involucrando árbitros europeos mostró que el porcentaje de grasa corporal de sus árbitros (11,3\%) son menores que de los árbitros americanos (Casajus \& Castagna, 2006). Estos valores son semejantes a los atletas del fútbol brasileño $(10,6 \%)$ (Guerra et al., 2004) e iguales a los jugadores de países de América del Sur (10,6) (Rienzi et al., 1998).

El primer artículo internacional, del que tenemos conocimiento, sobre el somatotipo de árbitros de fútbol, fue publicado por Fernández et al. (2008), involucrando árbitros chilenos. En está investigación los árbitros fueron clasificados como meso-endomorfos (predomino del tejito muscular sobre el tejido adiposo), misma clasificación de los árbitros asistentes de la CBF. Mientras que el grupo de la FPF tenía una característica endo-mesomorfo, o sea, predominio del tejido adiposo sobre el tejito muscular (Tabla II). En un análisis más detallado de las somatocartas (Fig. 1), observamos que existe una mayor concentración de los árbitros asistentes de la CBF, en la parte endomorfia de la somatocarta (73\%) a diferencia del FPF (50\%, Fig. 2), lo que contribuyó para el cambio en la clasificación de los mismos.
Debido a que existe una gran heterogeneidad de los datos, el cálculo de la DDS fue estadísticamente significativa (DDS=2,22), lo que confirma que los dos grupos tienen diferentes características en relación con el somatotipo. DDS permite verificar la distancia entre dos somatotipos, estableciendo que la diferencia es estadísticamente significativa cuando la DDS es >2 (Hebbelinck et al., 1975). Esto nos lleva a creer que el perfil atlético (cantidad de tejido adiposo) es un factor determinante en la elección de los árbitros que serán selecionados para el cuadro nacional.

En la Tabla III se encuentran los resultados referentes a la prueba que mide la velocidad (carrera de 50 metros) de los árbitros asistentes de la CBF y de la FPF. El análisis de los resultados, es decir, la suma de la primera con la segunda carrera, de los árbitros asistentes de la CBF y FPF, demuestran que los árbitros de la CBF son más veloces, siendo que esta diferencia es estadísticamente significativa $(\mathrm{p}=0,0001)$. El tiempo promedio encontrado en este estudio es similar a la de literatura científica en donde, por ejemplo Da Silva \& Rodrigues-Añez (2003), describen que cuando evaluaron árbitros de la CBF el tiempo promedio fue de $6,74 \pm 0,35$ s. Árbitros que participaron del concurso de la Union European Football Association (UEFA), para nuevos árbitros de la FIFA en 1995, presentaron un tiempo de 7,09 s (Eissmann, 1996), siendo que en un estudio reciente, árbitros españoles presentaron un tiempo promedio de 6,76 $\pm 0,25 \mathrm{~s}$ (Casajus \& Castagna). Mientras estos datos son de árbitros no de árbitros asistentes. En nuestra revision de la literatura científica encontramos dos estudios con datos de árbitros asistentes. El tiempo promedio de $6,9 \mathrm{~s}$ fue obtenido por árbitros daneses (Krustrup et al.). Fue descrito $6,86 \pm 0,12 \mathrm{~s}$, para árbitros asistentes en Brasil (Da Silva \& Rodrigues-Añez, 2003) y en un estudio reciente involucrando asistentes internacionales del cuadro de la FIFA, fue relatado un tiempo promedio de 6,89 \pm 0,35 s (Mallo et $a l$.). Los datos encontrados en esta investigación demuestran que los árbitros asistentes de Brasil presentan un rendimiento en los tests de 50 metros semejante a los árbitros 
internacionales. Según Weston et al., la capacidad física velocidad debe ser trabajada constantemente por los árbitros durante sus entrenamientos. De esta forma les permitirá estar más próximos del balón después de una jugada de contra-ataque en caso de tener que juzgar alguna incorrección o intervenir en la jugada.

El test de Cooper, que consiste en correr o andar ininterrumpidamente por 12 minutos, fue la última prueba a ser aplicada en los árbitros. El desplazamiento promedio durante este test obtenido por los dos grupos son exhibidos en la Tabla IV. Los resultados de esta investigación son semejantes al promedio presentado por los árbitros de brasileños de la $\mathrm{CBF}$ con 2883 metros (Da Silva \& Rodrigues-Añez, 2003) y árbitros internacionales, pues en un estudio realizado en Grecia, los árbitros de la División A tuvo un promedio de $2778 \pm 128$ metros durante la prueba de Cooper (Rontoyannis et al.) y de árbitros italianos promedio de $2866 \pm 164$ metros (Castagna et al.). Sin embargo, en un estudio publicado recientemente, los índices obtenidos por árbitros españoles en esta prueba fue mayor que el nuestro, es decir, $2.984 \pm 125$ metros en 12 minutos (Casajus \& Castagna). Los resultados de ambos gru- pos aquí estudiados, son también similar al rendimiento de árbitros asistentes de otros estudios. Da Silva \& RodriguesAñez (2003) que estudiaron árbitros asistentes de elite de Brasil, mencionan una distancia de 3030 metros recorrida por los asistentes durante los 12 minutos. Arbitros asistentes daneses presentaron un rendimiento promedio de 2889 metros (Krustrup et al.), mismo rendimiento (2962 metros) en un estudio reciente publicado con árbitros internacionales del cuadro de la FIFA (Mallo et al.).

Con el deseo de mejorar la apititud física de los árbitros la UEFA, la FIFA y más recientemente la Federación Inglesa buscó profesionalizar sus árbitros, pues, el fútbol mundial se tornó más competitivo y más rápido en los últimos años y el árbitro no puede permanecer a la margen de éste desarrollo (Weston et al.). Además de una buena preparación física para poder evaluar las jugadas, evitando con ésto que las reglas sean violadas, el árbitro debe también estar bien posicionado para visualizar las agresiones entre los atletas, pues el riesgo de un jugador sufrir una herida es cerca de 1000 veces mayor del encontrado en la mayoría de otras profesiones (Fuller et al., 2004).

SILVA, A. I. Somatotype and physical fitness of the assistant referees in soccer. Int. J. Morphol., 29(3):792-798, 2011.

SUMMARY: The aim of this study was to analyze the somatotype of assistant referees and examine its correlation with physical performance during FIFA physical tests. Study subjects were referees from the Brazilian Football Confederation (CBF) and Paranese Football Federation (FPF) who appeared before the Physical Evaluation Committee of the FPF in order to submit to the physical fitness tests. The sample was comprised of 11 referees from the CBF and considered the elite of Brazil, and 34 FPF referees who officiate at regional level in the state of Parana. In order to determine body composition nine skin folds, nine body circumferences and four bone diameter measures were used. To assess variables related to physical fitness a battery of the following FIFA physical tests were used: two runs of 50 meters and 12 minute running tests. Data analysis of body composition indicated fat percentage of assistant referees from the CBF was $16.74 \pm 3.71 \%$, and assistants of the FPF was $17.09 \pm 4.38 \%$. There were no statistically significant differences ( $p>0.05$ ). With regard to somatotype, CBF assistants were classified as mesoendomorph and FPF assistants as endo-mesomorph, in other words, $\mathrm{CBF}$ assistants group showed a higher predominant muscular component over fat. The difference between somatotypes was statistically significant (DDS=2.22). In reference to the speed test the CBF assistant referees were faster than the FPF referees, the difference being statistically significant between results $(\mathrm{p}=0.0001)$. During a match, assistant referees perform a lesser physical effort than the main referee; their performance however was similar to the main referee in the physical tests.

KEY WORDS: Referee; Soccer; Somatotype.

\section{REFERENCIAS BIBLIOGRÁFICAS}

Callaway, C. W.; Chumlea, W. C.; Bouchard, C.; Himes, J. H.; Martin, A. D.; Mitchell, C. D.; Mueller, W. H.; Roche, A. F. \& Seefeldt, V. D. Circumferences. In. Lohman, T. G.; Roche, A. F. \& Martorell, R. Anthropometric standardization reference manual. Champaign, Human Kinetics Books, 1991.

Casajus, J. A. \& Castagna, C. Aerobic fitness and field test performance in elite Spanish soccer referees of different ages. J. Sci. Med. Sport, 10(6):382-9, 2007.

Carter, J. E. Somatotypes of college football players. Res. Q., 39(3):476-81, 1968.
Carter, J. E. L. The heath-carter anthropometric somatotype instruction manual. San Diego, San Diego State University, 2002.

Carter, J. E. L. \& Heath, B.H. Somatotyping-Development and Applications. New York, Cambridge University Press, 1990.

Castagna, C.; Abt, G. \& D’Ottavio, S. Relation between fitness tests and match performance in elite Italian soccer referees. J. Strength Cond. Res., 16(2):231-5, 2002.

Da Silva, A. I. Bases científicas e metodológicas para o treinamento do árbitro de futebol. Curitiba, UFPR, 2005.

Da Silva, A. I. \& Fernandez, R. Dehydration of football referees during a match. Br. J. Sports Med., 37:502-6, 2003. 
Da Silva, A. I. \& Rech, C. R. Somatotipo e composição corporal de árbitros e árbitros assistentes da CBF. Rev. Bras. Cineantropom. Desempenho Hum., 10(2):143-8, 2008.

Da Silva, A. I. \& Rodriguez-Añez, C. R. Ações motoras do árbitro assistente de futebol durante a partida. Rev. Bras. Ciên. e Mov., 10(1):29-34, 2002.

Da Silva, A. I. \& Rodriguez-Añez, C. R. Níveis de aptidão física e perfil antropométrico dos árbitros de elite do Paraná credenciados pela Confederação Brasileira de Futebol (CBF). Rev. Port. Cien. Desp., 3(3):18-26, 2003.

De Rose, E. H., Pigatto, E. \& De Rose, R. C. F. Cineantropometria, educação física e treinamento desportivo. Rio de Janeiro, SEED/MEC, 1984.

Duarte, O. Futebol: história e regras. São Paulo, Ed. Makron Books, 1997.

Eissmann, H. J. El árbitro de fútbol. Madrid, Editorial Gymnos, 1996.

Fernández, V. G. E.; Da Silva, A. I. \& Arruda, M. Anthropometric Profile and Physical Fitness of the Professional Referees Chilean Soccer. Int. J. Morphol., 26(4):897-904, 2008.

FIFA. Reglas de juego. Zurich, 2010. Disponible en: http:// es.fifa.com/worldfootball/lawsofthegame/index.html

Fuller, C. W.; Junge, A. \& Dvorak, J. An assessment of football referees'decisions in incidents leading to player injuries. Am. J. Sports Med., 32(1 Suppl):17S-22S, 2004.

Guerra, I.; Chaves, R.; Barros, T. \& Tirapegui, J. The influence of fluid ingestion on performance of soccer players during a match. Journal of Sports Science and Medicine, 3:198-202, 2004.

Harrison, G. G.; Buskirk, E. R.; Carter, J. E. L.; Johnston, F. E.; Lohman, T. G.; Pollock, M. L.; Roche, A. F. \& Wilmore, J. H. Skinfold thicknesses and measurement technique. In: Lohman, T. G.; Roche. A. F. \& Martorell, R. (Eds.). Anthropometric standardization reference manual. Champaign, Human Kinetics Books, 1991.

Hebbelinck, M.; Carter, L. \& De Garay, A. Body build and somatotype of Olimpic swimmers, divers and water polo players. In: Lewillie, L. \& Clarys, J. P. Swimming. Baltimore, University Park Press, 1975. pp.285-305.

Jackson, A. S. \& Pollock, M. L. Generalized equations for predicting body density of men. Br. J. Nutr. 40(3):497-504, 1978.

Junior, A. T. C.; Cunha, A. C. P. T.; Schneider, A. T. \& Dantas, P. M. S. Características dermatoglíficas, somatotípicas, psicológicas e fisiológicas da seleção brasileira feminina adulta de handebol. Fit. Perform. J., 5(2):81-6, 2006.
Krustrup, P.; Mohr, M. \& Bangsbo, J. Activity profile and physiological demands of top-class soccer assistant refereeing in relation to training status. J. Sports Sci., 20(11):861-71, 2002.

Mallo, J.; Navarro, E.; García-Aranda, J. M.; Gilis, B. \& Helsen, W. Analysis of the kinematical demands imposed on top-class assistant referees during competitive soccer matches. J. Strength Cond. Res., 22(1):235-42, 2008.

Queiroga, M. R.; Ferreira, S. A. \& Romanzini, M. Perfil antropométrico de atletas de futsal feminino de alto nível competitivo conforme a função tática desempenhada no jogo. Rev. Bras. Cineantropom. Desempenho Hum., 7(1):30-4, 2005.

Rahmawati, N. T.; Budiharjo, S. \& Ashizawa, K. Somatotypes of young male athletes and non-athlete students in Yogyakarta, Indonesia. Anthropo. Sci., 115(1):1-7, 2006.

Rienzi, E.; Mazza. J. C.; Carter, J. E. L. \& Reilly, T. Futbolista Sudamericano de Elite: Morfología, Análisis del Juego y Performance. Rosario, Biosystem Servicio Educativo, 1998.

Rontoyannis, G. P.; Stalikas, A.; Sarros, G. \& Vlastaris, A. Medical, morphological and funcional aspects of Greek football referees. J. Sports Med. Phys. Fitness, 38(3):208-14, 1998.

Ross, W. D. \& Marfell-Jones, M. J. Cineantropometria. In: McdougaL, J. D.; Wenger, H. \& Green, H. J. Evaluación fisiologica del deportista. Barcelona, Editorial Paidotribo, 1995.

Siri, W. E. Body composition from fluid space and density. In: Brozek, J. \& Hanschel, A. Techniques for measuring body composition. Washington D.C., National Academy of Sciences, 1961. pp.223-4.

Weston, M.; Helsen, W.; MacMahon, C. \& Kirkendall, D. The impact of specific high-intensity training sessions on football referees' fitness levels. Am. J. Sports Med., 32(1 Suppl):54S61S, 2004.

Wilmore, J. H.; Frisancho, R. A.; Gordon, C. C.; Himes, J. H.; Martin, A. D.; Martorell, R. \& Seefeldt, V. D. Body Breadth Equipment and Measurement Techniques. In: Lohman, T. G.; Roche, A. F. \& Martorell, R. Anthropometric standardization reference manual. Champaign, Human Kinetics Books, 1991.

\section{Dirección para correspondencia: \\ Alberto Inácio da Silva \\ Universidade Estadual de Maringá \\ Campus Ivaiporã \\ Paraná \\ BRAZIL}

Email: albertoinacio@bol.com.br

Recibido : 24-11-2010

Aceptado: 01-04-2011 